The Astrophysical Journal, 661: L187-L190, 2007 June 1

(C) 2007. The American Astronomical Society. All rights reserved. Printed in U.S.A.

\title{
A PHOTOEVAPORATING ROTATING DISK IN THE CEPHEUS A HW2 STAR CLUSTER
}

\author{
I. Jiménez-Serra, ${ }^{1}$ J. Martín-Pintado, ${ }^{1}$ A. Rodríguez-Franco, ${ }^{1,2}$ C. Chandler,${ }^{3}$ C. Comito, ${ }^{4}$ And P. Schilke \\ Received 2007 March 1; accepted 2007 April 20; published 2007 May 11
}

\begin{abstract}
We present VLA and PdBI subarcsecond images $\left(\sim 0.15^{\prime \prime}-0.6^{\prime \prime}\right)$ of the radio continuum emission at $7 \mathrm{~mm}$ and of the $\mathrm{SO}_{2} J=19_{2,18} \rightarrow 18_{3,15}$ and $J=27_{8,20} \rightarrow 28_{7,21}$ lines toward the Cep A HW2 region. The $\mathrm{SO}_{2}$ images reveal the presence of a hot core internally heated by an intermediate-mass protostar, and a circumstellar rotating disk around the HW2 radio jet of size $600 \times 100 \mathrm{AU}$ and mass $\sim 1 M_{\odot}$. Keplerian rotation for the disk velocity gradient of $\sim 5 \mathrm{~km} \mathrm{~s}^{-1}$ requires a $9 M_{\odot}$ central star, which cannot explain the total luminosity observed in the region. This may indicate that the disk does not rotate with a Keplerian law due to the extreme youth of this object. Our high-sensitivity radio continuum image at $7 \mathrm{~mm}$ shows, in addition to the ionized jet, an extended emission to the west (and marginally to the south) of the HW2 jet, filling the southwest cavity of the HW2 disk. From the morphology and location of this free-free continuum emission at centimeter and millimeter wavelengths (spectral index $\sim 0.4-1.5$ ), we propose that the disk is photoevaporating due to the UV radiation from the central star. All this indicates that the Cep A HW2 region harbors a cluster of massive stars. Disk accretion seems to be the most plausible way to form massive stars in moderate density/luminosity clusters.
\end{abstract}

Subject headings: ISM: individual (Cepheus A) - ISM: molecules — stars: formation

\section{INTRODUCTION}

Massive stars (in excess of $\sim 8 M_{\odot}$ ) are known to form in clusters within the dense cores of giant molecular clouds (Garay \& Lizano 1999). The formation processes of these stars, however, still remain unclear. It has been proposed that massive stars form either through accretion of material from a circumstellar disk (McKee \& Tan 2003) or through the merging of several low-mass stars (Bonnell \& Bate 2002). Since the sites of massive star formation are located at distances of $\geq 0.5 \mathrm{kpc}$, subarcsecond observations are required to measure small-scale structures relevant to the star formation process.

Cepheus A East (at 725 pc; Johnson 1957) is a very active region of massive star formation (see, e.g., Hughes \& Wouterloot 1984; Torrelles et al. 1996; Garay et al. 1996; Gómez et al. 1999). The HW2 radio jet, the brightest radio source in the region (Hughes \& Wouterloot 1984), is known to power the large-scale molecular outflow seen in the northeast-southwest direction (Narayanan \& Walker 1996; Gómez et al. 1999). Perpendicular to the jet-outflow axis, Torrelles et al. (1996) inferred the presence of a rotating and contracting circumstellar disk (size $0.8^{\prime \prime}$, or $600 \mathrm{AU}$ ) from the spatial and velocity distribution of the water maser emission around the HW2 jet.

Patel et al. (2005) have recently reported a flattened $\mathrm{CH}_{3} \mathrm{CN}$ structure (beam $\sim 1^{\prime \prime}$ ) with size 1.6" ( 1200 AU), suggesting that all molecular emission around HW2 is located in a rotating disk. However, the detection of the first hot core associated with an intermediate-mass protostar in the vicinity of HW2 (Martín-Pintado et al. 2005) clearly contrasts with the idea of a single protostellar source undergoing disk accretion, as suggested by Patel et al. (2005). Furthermore, linear/arcuate water

\footnotetext{
${ }^{1}$ Departamento de Astrofísica Molecular e Infrarroja, Instituto de Estructura de la Materia (CSIC), C/Serrano 121, E-28006 Madrid, Spain; izaskun@ damir.iem.csic.es, martin@damir.iem.csic.es, arturo@damir.iem.csic.es.

${ }^{2}$ Escuela Universitaria de Óptica, Departamento de Matemática Aplicada (Biomatemática), Universidad Complutense de Madrid, Avda. Arcos de Jalón s/n, E-28037 Madrid, Spain.

${ }^{3}$ National Radio Astronomy Observatory P.O. Box O, Socorro NM 87801; cchandle@nrao.edu.

${ }^{4}$ Max Planck Institute for Radio Astronomy, Auf dem Hügel 69, D-53121 Bonn, Germany; ccomito@mpifr-bonn.mpg.de, schilke@mpifr-bonn.mpg.de.
}

maser structures (Torrelles et al. 2001) and high angular resolution radio continuum observations (Curiel et al. 2002) have revealed at least three different young stellar objects (YSOs) within a projected area of $\sim 0.6^{\prime \prime} \times 0.6^{\prime \prime}$, making the actual picture of the Cep A HW2 region even more complex.

The morphology of the ionized gas restricted to the radio jet in all directions, however, requires the presence of circumstellar material confining the UV photons emitted by the exciting source. In this Letter, we present subarcsecond $\left(\sim 0.15^{\prime \prime}-\right.$ $\left.0.6^{\prime \prime}\right)$ VLA and PdBI images that resolve for the first time the hot molecular gas around HW2 in a photoevaporating rotating disk around the radio jet and a nearby hot core. The hot core is an independent protostar in the cluster around HW2 that likely drives the east-west outflow found in the region.

\section{OBSERVATIONS AND RESULTS}

Observations of the $\mathrm{SO}_{2} J=19_{2,18} \rightarrow 18_{3,15}\left(E_{u}=183 \mathrm{~K}\right)$ line toward the Cep A HW2 region were carried out in 2005 April 12 with the Very Large Array (VLA) in the B configuration. We used the 2 IF (AD) spectral line mode with a bandwidth of 12.5 MHz and 32 channels per IF. The spectral resolution provided by the correlator was $390.625 \mathrm{kHz}\left(\sim 2.7 \mathrm{~km} \mathrm{~s}^{-1}\right.$ at $\left.7 \mathrm{~mm}\right)$. The central channel was set at $-10 \mathrm{~km} \mathrm{~s}^{-1}$ (Martín-Pintado et al. 2005). 3C 84 ( $9.00 \mathrm{Jy}), 3 \mathrm{C} 48$ ( $\sim 0.64 \mathrm{Jy})$, and $2250+558$ $(\sim 0.46 \mathrm{Jy})$ were used as bandpass, flux density, and phase calibrators, respectively. Calibration, continuum subtraction, (natural-weighted) imaging, and cleaning were carried out with AIPS. The $\mathrm{SO}_{2} J=27_{8,20} \rightarrow 28_{7,21}\left(E_{u}=505 \mathrm{~K}\right)$ line was observed between 2003 December and 2004 March with the IRAM Plateau de Bure Interferometer (PdBI) in the $\mathrm{AB}$ configuration. The final maps are $1.6 \mathrm{~km} \mathrm{~s}^{-1}$ channel width. We used $3 \mathrm{C} 454.3$ ( 7 Jy), NRAO 150 ( 3 Jy), and 2037+511 ( 0.6 Jy) as bandpass, flux density, and phase calibrators, respectively. Calibration, continuum subtraction, imaging, and cleaning were done with GILDAS.

In Figure 1 (upper panels), we show the $7 \mathrm{~mm}$ continuum map (beam of $0.18^{\prime \prime} \times 0.16^{\prime \prime}$, P.A. $=30^{\circ}$ ) obtained by averaging line-free channels, and the $\mathrm{SO}_{2} J=19_{2,18} \rightarrow 18_{3,15}$ line images at $-7.3,-10.0$, and $-12.7 \mathrm{~km} \mathrm{~s}^{-1}$ (beam of $\sim 0.35^{\prime \prime}$ ), obtained by tapering our data with a Gaussian function of $500 \mathrm{k} \lambda$. We 

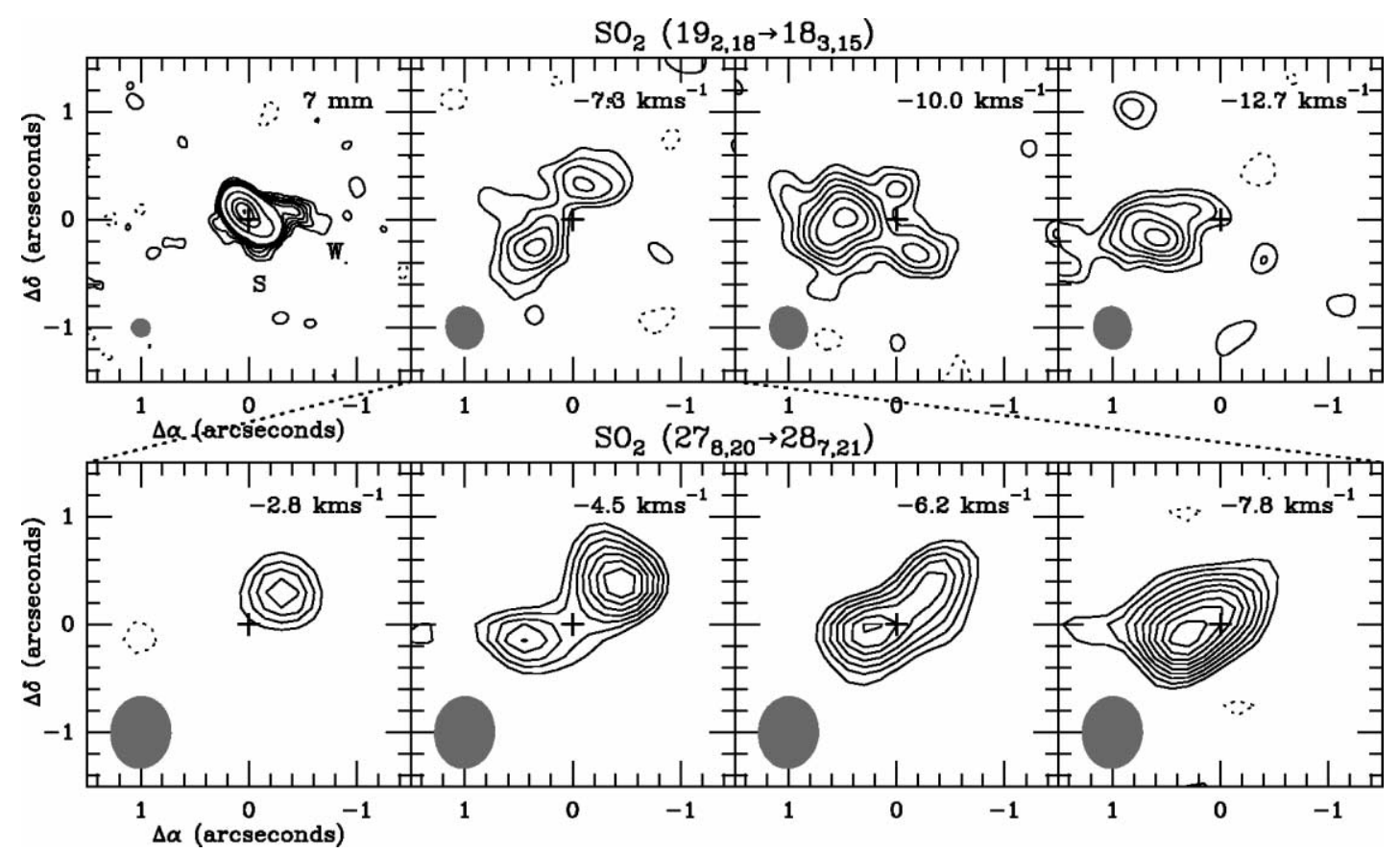

FIG. 1.-Upper panels: Radio continuum emission at $7 \mathrm{~mm}$ and $\mathrm{SO}_{2} J=19_{2,18} \rightarrow 18_{3,15}$ line images observed at $-7.3,-10.0$, and $-12.7 \mathrm{~km} \mathrm{~s}{ }^{-1}$ with the VLA. The contour levels for the continuum map are $-0.4,0.4(2 \sigma), 0.6,0.8,1.0,1.2,1.4,5,13,21$, and $29 \mathrm{mJy}^{\text {beam }}{ }^{-1}$, and for the $\mathrm{SO}_{2}$ images are $-3,2^{-1}$ $(2 \sigma), 3,4,5,6,7$, and $8 \mathrm{mJy}_{\text {beam }}^{-1}$. Lower panels: $\mathrm{SO}_{2} J=27_{8,20} \rightarrow 28_{7,21}$ line maps at $-2.8,-4.5,-6.2$, and $-7.8 \mathrm{~km} \mathrm{~s}^{-1}$ observed with the PdBI. The contour levels are $-3,3,4,5,6,7,8$, and $9 \times 14$ mJy beam ${ }^{-1} \mathrm{~km} \mathrm{~s}^{-1}$, the $1 \sigma$ noise level of the maps. Beam sizes are shown at lower left corner, and the reference position is $\alpha=22^{\mathrm{h}} 56^{\mathrm{m}} 17.98^{\mathrm{s}}, \delta=+62^{\circ} 01^{\prime} 49.5^{\prime \prime}(\mathrm{J} 2000)$

resolve the continuum emission in the strong thermal radio jet (deconvolved size of $0.3^{\prime \prime} \times 0.1^{\prime \prime}$ ), and a weak extended structure to the west, and marginally to the south, of HW2 (hereafter, the $\mathrm{W}$ and $\mathrm{S}$ components; see Fig. 1). The orientation of the radio jet $\left(\right.$ P.A. $\left.\simeq 46^{\circ}\right)$ is similar to that derived by Rodríguez et al. (1994). The peak continuum intensity is $27.8 \pm 0.2 \mathrm{mJy}$, and the integrated flux of the radio jet is $\sim 53 \mathrm{mJy}$, in agreement with the results of Curiel et al. (2006). The $\mathrm{W}$ component is detected at an $8 \sigma$ level, with a peak intensity of $1.2 \mathrm{mJy}$. This continuum emission is also barely observed at $3.6 \mathrm{~cm}$ (epochs 2000 and 2002; see Curiel et al. 2006) at a $2 \sigma$ level. The lack of detection of the $\mathrm{W}$ component in the $1.3 \mathrm{~cm}$ maps of Curiel et al. (2006) is consistent with a spectral index of $\sim 1.5$ (§ 3). Despite the smaller spatial extension of the $S$ component, this emission could be the counterpart of the VLA-R5 source seen at $3.6 \mathrm{~cm}$ (Curiel et al. 2002, 2006).

The $\mathrm{SO}_{2} J=19_{2,18} \rightarrow 18_{3,15}$ emission of Figure 1 (upper panels) reveals different features in the vicinity of the HW2 radio jet. The $-7.3 \mathrm{~km} \mathrm{~s}^{-1}$ channel map (integrated flux of $38 \mathrm{mJy}$ ) shows an elongated structure with an orientation (P.A. $\simeq-34^{\circ}$ ) nearly perpendicular to that of the radio jet and reminiscent of a disk. Images of the emission from several molecules show a chemical segregation of the hot cores around HW2 (Comito et al. 2007; Brogan et al. 2007), with the $\mathrm{SO}_{2}$ emission peaking toward the east of HW2. Our $\mathrm{SO}_{2}$ images, however, show that this molecule is clearly sampling a coherent spatial structure at both sides of the radio jet, as expected for a circumstellar disk around HW2. Furthermore, the higher sensitivity and velocity resolution PdBI images of the $\mathrm{SO}_{2} J=27_{8,20} \rightarrow 28_{7,21}$ line (lower panels in Fig. 1) show that the redshifted emission at $-2.8 \mathrm{~km} \mathrm{~s}^{-1}$ is located to the northwest of HW2, while the blueshifted emission at $-7.8 \mathrm{~km} \mathrm{~s}^{-1}$ is located to the southeast of the radio jet. This velocity gradient of $\sim 5 \mathrm{~km} \mathrm{~s}^{-1}$ is consistent with a rotating disk. Although the central velocity of the disk $\left(-5 \mathrm{~km} \mathrm{~s}^{-1}\right)$ is different from the systemic velocity of the cloud $\left(-10 \mathrm{~km} \mathrm{~s}^{-1}\right)$, it is not uncommon to find protostars with velocities different from that of the ambient cloud (e.g., Morris et al. 1980).

All the morphological and kinematical evidence therefore points toward a circumstellar rotating disk around HW2 with a deconvolved size of $0.8^{\prime \prime} \times 0.15^{\prime \prime}(600 \times 100 \mathrm{AU})$. This size is much smaller than that obtained by Patel et al. (2005) from the line emission, but similar to that measured in the continuum images. Although we cannot rule out the possibility that any substantial flaring could appear at smaller scales than our beam, the circumstellar disk around HW2 seems to be very thin.

The $\mathrm{SO}_{2} J=19_{2,18} \rightarrow 18_{3,15}$ channel map at $-10.0 \mathrm{~km} \mathrm{~s}^{-1}$ (Fig. 1) shows a compact condensation (size of $0.6^{\prime \prime}$ and integrated flux of $50 \mathrm{mJy}$ ) whose location ( 0.4" east of HW2) coincides with that of the hot core heated by an intermediate-mass protostar reported by Martín-Pintado et al. (2005). For the $-12.7 \mathrm{~km} \mathrm{~s}^{-1}$ channel (Fig. 1), we find elongated $\mathrm{SO}_{2}$ emission in the southeastnorthwest direction (size of $\sim 0.7^{\prime \prime} \times 0.4^{\prime \prime}$, P.A. $=-72^{\circ}$, and integrated flux of $35 \mathrm{mJy}$ ) whose emission peak is located $\sim 0.7^{\prime \prime}$ (500 AU) east of HW2, suggesting that this emission is not associated with the hot core.

\section{THE DISK AROUND THE HW2 RADIO JET}

Figure 2 shows the superposition of the $\mathrm{SO}_{2} J=19_{2,18} \rightarrow$ $18_{3,15}-7.3 \mathrm{~km} \mathrm{~s}^{-1}$ channel map (thin contours and gray scale) on the radiocontinuum images (thick solid contours) at $7 \mathrm{~mm}$ (upper panel) and $3.6 \mathrm{~cm}$ (lower panel; Curiel et al. 2006). The morphology of the $\mathrm{SO}_{2}$ emission shows that the circumstellar molecular disk confines the HW2 radio jet. The location of the central source (see filled stars in Fig. 2; Curiel et al. 2006) is very close to the geometrical center of the disk and to the peak of the continuum emission at $7 \mathrm{~mm}$.

The water vapor masers reported by Torrelles et al. (1996) are distributed along the $\mathrm{SO}_{2}$ emission (Fig. 2), supporting the 


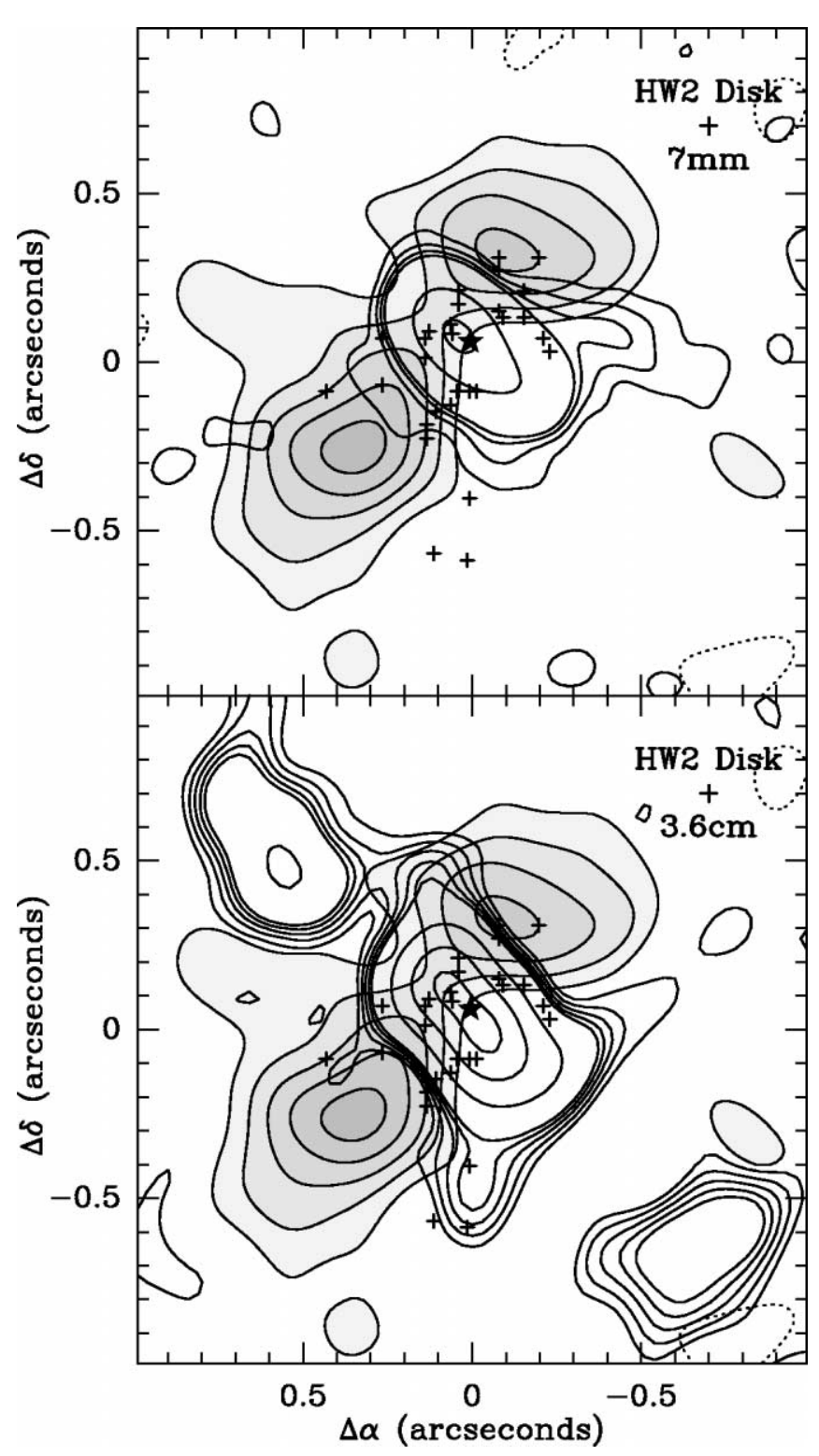

FIG. 2.- $\mathrm{SO}_{2}$ emission map at $-7.3 \mathrm{~km} \mathrm{~s}^{-1}$ (thin contours and gray scale) superimposed on our $7 \mathrm{~mm}$ continuum image (thick contours, upper panel) and on the $3.6 \mathrm{~cm}$ continuum map of Curiel et al. (2006) for the 2000 epoch (thick contours, lower panel). Contour levels for the $7 \mathrm{~mm}$ map are $-0.4,0.4$ $(2 \sigma), 0.8,1.2,1.6,10$, and $26 \mathrm{mJy}_{\mathrm{beam}}^{-1}$, and for the $3.6 \mathrm{~cm}$ image are 0.08 $(2 \sigma), 0.12,0.16,0.20,0.24,0.64,1.28$, and $1.92 \mathrm{mJy}$ beam $^{-1}$. Contour levels for the $\mathrm{SO}_{2}$ emission are as in Fig. 1. Crosses mark the positions of the water masers (Torrelles et al. 1996). Filled stars show the location of the powering source of the HW2 jet (Curiel et al. 2006).

idea of a rotating disk around the HW2 ionized jet. Since the $\mathrm{H}_{2} \mathrm{O}$ maser emission is located at the interface between the radio jet and the HW2 disk, the kinematics of the water masers are likely dominated by the jet-disk interaction. In particular, the R4 arcuate maser structure (Torrelles et al. 2001) traces the edge of the disk as if the masers were located on its surface. The R1, R2, and R3 linear maser structures delineate the northwest edge of the radio jet, suggesting that the ionized jet is generating a cavity as it propagates through the molecular gas (Torrelles et al. 2001). The submillimeter water masers tracing the hot gas (Patel et al. 2007) appear at the inner region of the disk.

Figure 2 also shows that the $\mathrm{W}$ and $\mathrm{S}$ continuum features clearly fill and surround the southwest cavity of the HW2 disk.
By comparing our $7 \mathrm{~mm}$ continuum image with that of Curiel et al. (2006) at $3.6 \mathrm{~cm}$ (Fig. 2), we estimate the spectral index $\alpha\left(S_{\nu} \propto \nu^{\alpha}\right)$ of the $\mathrm{W}$ and $\mathrm{S}$ components. Using the $3.6 \mathrm{~cm}$ peak flux of Figure 2, we derive a spectral index of 1.5 for the $\mathrm{W}$ component. From the $3 \sigma$ level noise of our $7 \mathrm{~mm}$ map, an upper limit for the spectral index of $\leq 0.4$ is also derived for the VLA-R5 continuum source, consistent with that previously obtained by Curiel et al. (2002). As discussed in $\S 5$, this freefree continuum emission may be explained by the photoevaporation of the HW2 disk by the UV radiation of the central source.

We can make a rough estimate of the mass of the disk by considering the integrated $\mathrm{SO}_{2}$ emission measured at -7.3 $\mathrm{km} \mathrm{s}^{-1}$. Assuming an excitation temperature of $\sim 160 \mathrm{~K}$ (see below and Martín-Pintado et al. 2005), the $\mathrm{SO}_{2}$ column density within the disk, given by

$$
N\left(\mathrm{SO}_{2}\right)=1.4 \times 10^{7} \frac{\nu(\mathrm{GHz})^{2}}{A_{u l} g_{u}} \int T_{B} d v\left(\mathrm{~K} \mathrm{~km} \mathrm{~s}^{-1}\right),
$$

is $\sim 6 \times 10^{18} \mathrm{~cm}^{-2}$. If we now consider an edge-on disk with a radius of $R=300 \mathrm{AU}$ and a height of $h=100 \mathrm{AU}$, the disk mass can be estimated as

$$
M_{d}=6 \times 10^{-31} R(\mathrm{AU}) h(\mathrm{AU}) N\left(\mathrm{SO}_{2}\right) \chi\left(\mathrm{SO}_{2}\right)^{-1} M_{\odot} .
$$

For a $\mathrm{SO}_{2}$ abundance of $\sim 10^{-7}$ (Charnley 1997), the disk mass would be $\sim 1 M_{\odot}$. Also, assuming Keplerian rotation for the velocity gradient of the disk $\left[\Delta v \sim 5 \mathrm{~km} \mathrm{~s}^{-1}\right.$ and $M_{*}=$ $\left.1.25 \times 10^{-3}\left(\Delta v / \mathrm{km} \mathrm{s}^{-1}\right)^{2}(R / \mathrm{AU}) M_{\odot}\right]$, we derive a binding mass of the central source of $M_{*} \sim 9 M_{\odot}$ (B2-type star), which is a factor of 2 smaller than that obtained by Patel et al. (2005).

\section{HOT CORE AND SHOCKED OUTFLOWING GAS}

The $\mathrm{SO}_{2} J=19_{2,18} \rightarrow 18_{3,15}$ emission at $-10 \mathrm{~km} \mathrm{~s}^{-1}$ (Fig. 1) resolves the hot core located $0.4^{\prime \prime}$ east of the HW2 jet. From the line peak flux at $-10 \mathrm{~km} \mathrm{~s}^{-1}$, and using the size of $\sim 0.6^{\prime \prime}$ and a linewidth of $\sim 5 \mathrm{~km} \mathrm{~s}^{-1}$ (Martín-Pintado et al. 2005), we find that the upper level $\mathrm{SO}_{2}$ column density averaged in a beam of $24^{\prime \prime}$ is $N_{u} / g_{u} \sim 2 \times 10^{11} \mathrm{~cm}^{-2}$. This $\mathrm{SO}_{2}$ column density is fully in agreement with an excitation temperature of $\sim 160 \mathrm{~K}$ (see the $\mathrm{SO}_{2}$ population diagram in Martín-Pintado et al. 2005), as expected if this emission traced the hot core associated with an intermediatemass protostar.

The $-12.7 \mathrm{~km} \mathrm{~s}^{-1} \mathrm{SO}_{2}$ emission (Fig. 1) is located 0.3" east from the hot core. As discussed in $\S 5$, this displacement, plus the spatially extended morphology and orientation of this emission, suggests a close association with shocked gas of one of the outflows in the region.

\section{DISCUSSION}

The subarcsecond VLA and PdBI images of the high- $J \mathrm{SO}_{2}$ lines have revealed the presence of a hot core heated by a YSO and a rotating disk around the $\mathrm{HW} 2$ jet. Furthermore, the $7 \mathrm{~mm}$ and $3.6 \mathrm{~cm}$ radio continuum images show free-free emission at the southwest cavity of the disk, suggesting that it is photoevaporating (Hollenbach et al. 1994). The derived spectral index of this emission $(\alpha \sim 0.4-1.5)$ is similar to those obtained by Jaffe \& Martín-Pintado (1999) for the broad recombination line objects that were proposed to be associated with photoevaporating disks. Most of these objects are very distant, making it difficult to resolve the neutral disk and the ionized flow produced 
by the photoevaporation. So far, only MWC 349 has been studied in some detail from recombination line masers (Martín-Pintado 2002). However, MWC 349 is a rather evolved object, which does not show the highly collimated jet but only the low-velocity outflow. If confirmed, the HW2 system would offer an unique opportunity to study the early phase of the ionized flow generated by photoevaporation of disks.

Our estimate for the mass of the disk $\left(\sim 1 M_{\odot}\right)$ is consistent with that obtained by Patel et al. (2005). This high disk mass could a priori contrast with those derived for early B-type stars $\left(\sim 0.2 M_{\odot}\right.$; Natta et al. 2000). However, since disk material is rapidly dispersed by photoevaporation in Herbig Be stars (Fuente et al. 2001), the detection of a massive disk around HW2 is in agreement with an object at an early stage of its evolution.

For the central source, however, we note that a B2-type star, as inferred from the Keplerian rotation, will provide a luminosity of $\sim 5 \times 10^{3} L_{\odot}$, which is only $25 \%$ the total IR luminosity measured in the Cep A HW2 region, believed to be dominated by the HW2 powering source $\left(\sim 2 \times 10^{4} L_{\odot}\right.$; Evans et al. 1981). Furthermore, the rate of ionizing photons derived for the central star is consistent with a B0.5 star with $\sim 20 M_{\odot}$. Recent non-Keplerian disk models show that small deviations from the Keplerian rotation in a disk at the early stages of its formation properly fit the molecular and continuum emission observed in low-mass stars, avoiding underestimation of the central source mass due to assuming Keplerian rotation (Piétu et al. 2005). Non-Keplerian rotation could be the explanation of the relatively small mass derived for the powering source of the HW2 jet.

Our images show that the hot core is an independent object located in the vicinity of HW2. In addition to the hot core, we have also detected low-velocity (blueshifted) $\mathrm{SO}_{2}$ emission $0.3^{\prime \prime}$ east of the hot core. High angular interferometric $\mathrm{SiO}$ images have shown low-velocity gas (red- and blueshifted) in the surroundings of the hot core along the southeast-northwest direc- tion (Comito et al. 2007). In particular, the location of the lowvelocity $\mathrm{SO}_{2}$ emission coincides with the blue lobe of the small-scale $\mathrm{SiO}$ outflow (Comito et al. 2007), supporting the idea that this $\mathrm{SO}_{2}$ emission is shocked gas associated with the east-west outflow seen in CO (Narayanan \& Walker 1996) and likely driven by the protostar associated with the hot core.

The detection of a circumstellar disk around the HW2 radio jet in a cluster of massive stars favors the hypothesis of accretion of material through a circumstellar disk for the formation of these objects (McKee \& Tan 2003). This is expected, since the Cep A HW2 region is a relatively low-density cluster. However, in high-density clusters, it may not be unlikely that massive stars form through the coalescence of low-mass stars, as proposed by Bonnell \& Bate (2002).

In summary, our subarcsecond images have resolved for the first time the hot gas surrounding the Cep A HW2 massive star cluster. A rotating disk of radius $300 \mathrm{AU}$ and a mass of $1 M_{\odot}$ is photoevaporating by the central UV photons of the powering source of the HW2 radio jet. Our VLA images also resolve the nearby hot core associated with an intermediate-mass star. This object is independent of the central source of the HW2 jet, and powers the east-west outflow found in the region. The formation of the most massive star in moderate-luminosity clusters may be produced through accretion disks.

We thank S. Curiel for kindly providing the radio continuum image at $3.6 \mathrm{~cm}$ shown in Figure 2. We are grateful to the IRAM staff in Grenoble, in particular J. M. Winters, for their support in the data calibration process. We also acknowledge the Spanish MEC for the support provided through projects number ESP2004-00665, AYA2003-02785-E, and "Comunidad de Madrid" government under PRICIT project S-0505/ ESP-0277 (ASTROCAM).

\section{Facilities: VLA, PdBI}

\section{REFERENCES}

Bonnell, I. A., \& Bate, M. R. 2002, MNRAS, 336, 659

Brogan, C. L., Chandler, C. J., Hunter, T. R., Shirley, Y. L., \& Sarma, A. P. 2007, ApJ, 660, L133

Charnley, S. B. 1997, ApJ, 481, 396

Comito, C., Schilke, P., Endesfelder, U., Jiménez-Serra, I., \& Martín-Pintado, J. 2007, A\&A, submitted (astro-ph/0704.1617)

Curiel, S., et al. 2002, ApJ, 564, L35 2006, ApJ, 638, 878

Evans, N. J., II, et al. 1981, ApJ, 244, 115

Fuente, A., Neri, R., Martín-Pintado, J., Bachiller, R., Rodríguez-Franco, A., \& Palla, F. 2001, A\&A, 366, 873

Garay, G., \& Lizano, S. 1999, PASP, 111, 1049

Garay, G., Ramírez, S., Rodríguez, L. F., Curiel, S., \& Torrelles, J. M. 1996, ApJ, 459, 193

Gómez, J. F., Sargent, A. I., Torrelles, J. M., Ho, P. T. P., Rodríguez, L. F., Cantó, J., \& Garay, G. 1999, ApJ, 514, 287

Hollenbach, D., Johnstone, D., Lizano, S., \& Shu, F. 1994, ApJ, 428, 654

Hughes, V. A., \& Wouterloot, J. G. A. 1984, ApJ, 276, 204

Jaffe, D. T., \& Martín-Pintado, J. 1999, ApJ, 520, 162

Johnson, H. L. 1957, ApJ, 126, 121
Martín-Pintado, J. 2002, in IAU Symp. 206, Cosmic Masers, ed. V. Mineese \& M. Reid (San Francisco: ASP), 226

Martín-Pintado, J., Jiménez-Serra, I., Rodríguez-Franco, A., Martín, S., \& Thum, C. 2005, ApJ, 628, L61

McKee, C., \& Tan, J. 2003, ApJ, 585, 850

Morris, M., Palmer, P., \& Zuckerman, B. 1980, ApJ, 237, 1

Narayanan, G., \& Walker, C. K. 1996, ApJ, 466, 844

Natta, A., Grinin, V. P., \& Mannings, V. 2000, in Protostars and Planets IV, ed. V. Mannings, A. Boss, \& S. S. Russell (Tucson: Univ. Arizona Press), 559

Patel, N. A., Curiel, S., Zhang, Q., Sridharan, T. K., Ho, P. T. P., \& Torrelles, J. M. 2007, ApJ, 658, L55

Patel, N. A., et al. 2005, Nature, 437, 109

Piétu, V., Guilloteau, S., \& Dutrey, A. 2005, A\&A, 443, 945

Rodríguez, L. F., Garay, G., Curiel, S., Ramírez, S., Torrelles, J. M., Gómez, Y., \& Velázquez, A. 1994, ApJ, 430, L65

Torrelles, J. M., Gómez, J. F., Rodríguez, L. F., Curiel, S., Ho, P. T. P., \& Garay, G. 1996, ApJ, 457, L107

Torrelles, J. M., et al. 2001, ApJ, 560, 853 